\title{
Chromosome-level Genome Assembly Provides Insight Into the Evolution of Chromosomes and the Globin Gene Superfamily in Gymnocypris Eckloni
}

\section{Fayan Wang}

Qinghai University

Lihan Wang

Qinghai University

Dan Liu

Qinghai University

Qiang Gao

Qinghai University

Miaomiao Nie

Qinghai University

Shihai Zhu

Qinghai University

Yan Chao

Qinghai University

Xi Yang

Qinghai University

Chaojie Yang

Qinghai University

Cunfang Zhang

Qinghai University

Rigui Yi

Qinghai University

Weilin Ni

Qinghai University

Guangxin Li

Qinghai University

Qichang Chen

Qinghai University

Fei Tian

Northwest Institute of Plateau Biology, Chinese Academy of Sciences

Kai Zhao 
Northwest Institute of Plateau Biology, Chinese Academy of Sciences

Delin Qi ( $\square$ delinqi@126.com )

Qinghai University

\section{Research Article}

Keywords: Gymnocypris eckloni, chromosome-level genome assembly, whole-genome duplication, chromosome evolution, globin gene superfamily

Posted Date: January 19th, 2022

DOI: https://doi.org/10.21203/rs.3.rs-1227329/v1

License: (a) This work is licensed under a Creative Commons Attribution 4.0 International License. Read Full License 


\section{Abstract}

Background: Gymnocypris eckloni is widely distributed in isolated lakes and the upper reaches of the Yellow River and is well adapted to the Qinghai-Tibetan plateau (QTP) aqueous environment.

Results: Here, we report the chromosome-level genome of G. eckloni, which is the first genome reported for this highly specialized schizothoracine fish. The final genome was assembled into $918.68 \mathrm{Mb}$ sequences using PacBio long-read and Illumina short-read sequences, including 711 scaffolds with a contig N50 length of $4.19 \mathrm{Mb}$ and a scaffold N50 length of $43.54 \mathrm{Mb}$. The assembled sequences were further anchored and orientated onto 23 pseudo-chromosomes. In total, 23,157 genes were annotated, representing $94.80 \%$ of the total predicted protein-coding genes. We conducted comparative genomic analyses and found that species-specific and rapidly expanding gene families in $G$. eckloni are mainly involved in Dorso-ventral axis formation, $A B C$ transporters, Peroxisome and Herpes simplex virus 1 infection. The phylogenetic analysis showed that $G$. eckloni was most closely related to $C$. carpio with an estimated divergence time of $\sim 34.8$ million years ago (Ma), which coincides with the first and second tectonic uplifts of the QTP. We also found that $G$. eckloni underwent an additional whole-genome duplication (WGD) event (4R) at 14.2 Ma, which had a significant effect on the evolution of chromosomes and the globin gene superfamily in G. eckloni, thereby facilitating the adaptation of $G$. eckloni to the plateau water environment.

Conclusions: The high-quality genome assembly of $G$. eckloni will serve as a valuable genomic resource for future research on the evolution and ecology of the schizothoracine fish.

\section{Background}

The Qinghai-Tibetan plateau (QTP) is characterized by high altitude, low oxygen partial pressure (hypoxia), low temperatures, dramatic temperature fluctuations, and high UV radiation. It is the highest and one of the youngest plateaus on Earth. Schizothoracine fish (Teleostei: Cyprinidae) are the largest and most diverse taxon within the QTP ichthyofauna [1, 2]. These fish have been accepted as ideal models for studying the molecular mechanisms underlying the adaptation to harsh environments [3-5]. Previous studies have shown that the karyotypes of Schizothoracinae range from 90 to \pm 446 and that almost all species were polyploid [6-9]. A recent genomic study confirmed that Schizothorax o'connori of Schizothoracinae was a young tetraploid that underwent a fourth whole-genome duplication (4R WGD) after the teleost-specific third WGD (3R WGD) [10]. Other studies indicated that the globin gene superfamily, toll-like receptor family, and interferon regulatory factors in a representative species from this subfamily underwent adaptive evolution in response to the plateau environment, specifically gene loss, and gain events as a result of genome and/or gene duplications [5, 11-13]. Gymnocypris eckloni is a representative species of the highly specialized schizothoracine fish that is widely distributed in isolated lakes and the upper reaches of the Yellow River, and is very well adapted to the plateau's aqueous environment [1, 2]. Investigating the genomic evolution of $G$. eckloni may shed light on the underlying molecular mechanisms involved in high-altitude adaptations in schizothoracine fish of the QTP. 
In the present study, we integrated PacBio long-read sequencing, Illumina short-read sequencing, and high-throughput chromosome conformation capture ( $\mathrm{Hi}-\mathrm{C})$ technology to generate a high-quality chromosome-level reference genome for $G$. eckloni. Comparative analyses with 13 other vertebrate genomes were conducted, focusing particularly on phylogenetic relationships, divergence time, and the expansion and contraction of gene families. Then, the G. eckloni WGD event (4R) was determined, and the evolution of chromosomes and the globin gene superfamily in G. eckloni were analyzed. The reference genome obtained in this study will provide a foundation for future investigations on the evolution and adaptation of schizothoracine fish.

\section{Results And Discussion}

\section{Genome sequencing and assembly of G. eckloni}

A total of $312.2 \mathrm{~Gb}$ PacBio reads, $215.7 \mathrm{~Gb}$ Illumina short reads, and $253.7 \mathrm{~Gb} \mathrm{Hi}-\mathrm{C}$ reads were generated in a female G. eckloni (Supplementary Fig. 1, Additional file 1; Supplementary Table 1, Additional file 2). After filtering, $215.2 \mathrm{~Gb}(231.2 \times$ coverage) of clean Illumina data were retained to perform a genome survey (Supplementary Table 1, Additional file 2). Based on the k-mer $(k=17)$ depth frequency distribution analysis, the genome size of $G$. eckloni was estimated to be $927.13 \mathrm{Mb}$ (Supplementary Fig. 2, Additional file 1; Supplementary Table 2, Additional file 2). For genome assembly, a total of $239 \mathrm{~Gb}$ clean PacBio long reads (334.6× coverage) with an average length of 23,706 bp were generated (Supplementary Table 1, Additional file 2). The data were assembled using wtdbg2 followed by Quiver and Pilon polishing using the $215.2 \mathrm{~Gb}$ (231.2× coverage) of Illumina HiSeq clean reads, which produced a $918.45 \mathrm{Mb}$ genome assembly with a contig N50 size of 4.19 Mb (Supplementary Table 3, Additional file 2). The assembled genome size was slightly larger than most other teleosts ( $700 \mathrm{Mb})[14,15]$, but obviously smaller than the genomes of Cyprinus carpio (1.83 Gb) and Carassius auratus (1.85 Gb) [16, 17]. The assembled sequences were further anchored and orientated onto 23 pseudo-chromosomes using $\mathrm{Hi}-\mathrm{C}$ data. The 23 pseudo-chromosomes ranged in size from 15.91 to $89.39 \mathrm{Mb}$ (Fig. 1a, Table 1), covering $\sim 98.52 \%$ of the whole genome. The heatmap of the pseudo-chromosome crosstalk confirmed the high quality of our genome assembly (Fig. 1a, b). Finally, the G. eckloni genome was obtained with 711 scaffolds and a total length of $918,681,488 \mathrm{bp}$, a contig N50 of $4.19 \mathrm{Mb}$, and scaffold N50 of 43.54 Mb (Supplementary Table 3, Additional file 2). 
Table 1

Statistics of the chromosome assemblies using Hi-C data.

\begin{tabular}{|c|c|c|c|}
\hline Pseudo-Chromosomes & Sequence IDs & Cluster Numbers & Sequences Lengths \\
\hline Chr01 & Hic_asm_0 & 170 & $44,161,858$ \\
\hline ChrO2 & Hic_asm_1 & 20 & $25,669,045$ \\
\hline Chr03 & Hic_asm_2 & 102 & $34,715,927$ \\
\hline ChrO4 & Hic_asm_3 & 125 & $61,913,669$ \\
\hline Chr05 & Hic_asm_4 & 6 & $28,665,261$ \\
\hline Chr06 & Hic_asm_5 & 66 & $43,543,958$ \\
\hline Chr07 & Hic_asm_6 & 154 & $61,756,641$ \\
\hline Chr08 & Hic_asm_7 & 118 & $47,622,867$ \\
\hline Chr09 & Hic_asm_8 & 62 & $27,783,661$ \\
\hline Chr10 & Hic_asm_9 & 69 & $32,896,108$ \\
\hline Chr11 & Hic_asm_10 & 82 & $21,739,851$ \\
\hline Chr12 & Hic_asm_11 & 29 & $32,200,481$ \\
\hline Chr13 & Hic_asm_12 & 42 & $29,129,546$ \\
\hline Chr14 & Hic_asm_13 & 56 & $28,882,651$ \\
\hline Chr15 & Hic_asm_14 & 232 & $70,525,535$ \\
\hline Chr16 & Hic_asm_15 & 195 & $89,391,071$ \\
\hline Chr17 & Hic_asm_16 & 193 & $16,898,605$ \\
\hline Chr18 & Hic_asm_17 & 54 & $15,913,822$ \\
\hline Chr19 & Hic_asm_18 & 68 & $33,789,651$ \\
\hline Chr20 & Hic_asm_19 & 190 & $46,494,833$ \\
\hline Chr21 & Hic_asm_20 & 100 & $32,874,627$ \\
\hline Chr22 & Hic_asm_21 & 96 & $39,527,105$ \\
\hline Chr23 & Hic_asm_22 & 101 & $39,007,358$ \\
\hline
\end{tabular}

We conducted a sequence consistency assessment using BWA software. The results showed that $93.40 \%$ of the reads could be mapped, covering $96.34 \%$ of the assembled genome when the lllumina short reads were mapped to the assembled genome (Supplementary Table 4, Additional file 2). The completeness of 
the final assembled genome was assessed using CEGMA and BUSCO analyses. The CEGMA analysis revealed that 221 conserved genes (89.11\% of the core eukaryotic genes) supported the completeness of the assembled genome (Supplementary Table 5, Additional file 2). The BUSCO analysis showed that $88.4 \%$ (single-copy genes: $82.3 \%$, duplicated genes: $6.1 \%$ ) of the 2,586 single-copy genes were identified as complete, $2.4 \%$ were fragmented, and $9.2 \%$ were missing from the assembled genome (Supplementary Table 6, Additional file 2). Overall, these analyses suggested that we assembled a high quality and chromosome-level genome of G. eckloni.

\section{Genome annotation}

The repeat sequences prediction analysis revealed that $47.63 \%$ of the G. eckloni genome was annotated as repetitive elements (Supplementary Table 7, Additional file 2), of which LTRs were the most abundant with a total length of $356.79 \mathrm{Mb}$, accounting for $38.84 \%$ of the whole genome. SINEs were the rarest with a total length of $2.37 \mathrm{Mb}$ and represented $0.26 \%$ of the whole genome (Supplementary Table 8, Additional file 2).

Using a comprehensive strategy based on homologous sequence searches, ab initio gene predictions, and RNA-seq-derived evidence from nine tissue and blood samples, a total of 24,430 protein-coding genes were predicted in the genome of $G$. eckloni. The average transcript length was 1,530.87 bp with an average coding sequence (CDS) length of $1,536.71 \mathrm{bp}$. The average exon number per gene was 8.88 with an average exon length of $173.00 \mathrm{bp}$ and average intron length of $1,862.69 \mathrm{bp}$ (Supplementary Fig. 3 , Additional file 1; Supplementary Table 9, Additional file 2). The comparison of gene numbers, average coding sequence lengths, and average gene lengths with eight other fish species indicated that our annotations were comprehensive (Supplementary Fig. 3, Additional file 1; Supplementary Table 10, Additional file 2).

A total of 23,157 genes were annotated using at least one public database and represented $94.80 \%$ of the total predicted protein-coding genes (Table 2). The functional annotations found that 20,432 (83.60\%), $23,110(94.60 \%)$, and 21,539 (88.20\%) genes had significant hits with proteins catalogued in Swissprot, NR, and InterPro, respectively. A total of 15,281 (62.60\%), 20,593 (84.30\%), and 19,290 (79.00\%) genes were annotated to GO, KEGG, and Pfam, respectively (Supplementary Fig. 4, Additional file 1; Table 2). Additionally, 1,717 miRNAs, 12,157 tRNAs, 1,780 rRNAs, and 1,152 snRNAs were identified, which had average lengths of $116.58,75.00,178.65$, and 133.32 bp, respectively (Supplementary Table 11, Additional file 2). Taken together, these analyses suggested a satisfying level of completeness and accuracy of genome annotation. 
Table 2

The number of genes with functional

classifications in G. eckloni.

\begin{tabular}{|lll|}
\hline Database & Number & Percent (\%) \\
\hline SwissProt & 20,432 & 83.60 \\
NR & 23,110 & 94.60 \\
KEGG & 20,593 & 84.30 \\
\hline InterPro & 21,539 & 88.20 \\
\hline GO & 15,281 & 62.60 \\
\hline Pfam & 19,290 & 79.00 \\
\hline Annotated & 23,157 & 94.80 \\
\hline Unannotated & 1,273 & 5.20 \\
\hline Total & 24,430 & 94.80 \\
\hline
\end{tabular}

Gene family analysis and phylogenetics of G. eckloni

To examine G. eckloni evolution, we conducted a comparative genomics analysis using the G. eckloni genome obtained from this study and 13 other vertebrate genomes downloaded from the Ensembl database (Astyanax mexicanus, Ictalurus punctatus, Danio rerio, C. carpio, Ctenopharyngodon idella, Oreochromis niloticus, Oryzias latipes, Takifugu rubripes, Gallus gallus, Homo sapiens, Mus musculus, Xenopus tropicalis, and Petromyzon marinus). A total of 24,619 gene families were identified among the 14 species (Supplementary Fig. 5, Additional file 1; Supplementary Table 12, Additional file 2), of which 2,739 core gene families were shared by all 14 species (Supplementary Fig. 6, Additional file 1). Compared to other species, we found 1,488 G. eckloni-specific genes, classified into 856 gene families. Further analysis revealed that 210 genes of the species-specific genes were significantly enriched five KEGG pathways, including Dorso-ventral axis formation, Peroxisome, $A B C$ transporters, Endocytosis and Herpes simplex virus 1 infection.

A phylogenetic tree was constructed using 597 single-copy orthologs with one-to-one correspondence in the different genomes, indicating that G. eckloni clustered together with C. carpio (Supplementary Fig. 7, Additional file 1). According to the time-calibrated phylogeny, the age of the most recent common ancestor (MRCA) of the teleost fish was estimated to be 211.8-254.1 Ma. The G. eckloni with the closest relationship to $C$. carpio shared an MRCA at $34.8 \mathrm{Ma}$ (26.0-41.4, Fig. 2a). Fluctuations in the ecogeographical environment and major hydrographic formation occurred during uplifts of the QTP and are generally accepted to have significantly affected speciation events of schizothoracine fish $[2,18]$. Our estimated divergence time for $G$. eckloni and $C$. carpio is similar to those obtained for other speciation 
events of congeners in family Cyprininae $[19,20]$, which is consistent with the first and second tectonic uplifts of the QTP [21].

Analysis of the expansion and contraction of the gene families revealed that there were 464 (1650 genes) expanded and 743 (192 genes) contracted gene families in G. eckloni when compared to its MRCA (Fig. 2a). Further functional enrichment analysis of the expanded gene families highlighted 62 significantly enriched GO terms $(p<0.05)$ and 25 KEGG pathways $(p<0.05)$ (Supplementary Table 13 , Additional file 3), while the contracted gene families highlighted 43 significant $\mathrm{GO}$ terms $(p<0.05)$ and 23 KEGG pathways $(p<0.05)$ (Supplementary Table 13, Additional file 3 ). The 25 KEGG pathways, including 625 genes from these expanded gene families, revealed that they were mainly classified as ABC transporters, Peroxisome, Herpes simplex virus 1 infection, Staphylococcus aureus infection, Axon guidance, Dorso-ventral axis formation, Pertussis, Legionellosis, Rap1 signaling pathway and so on (Supplementary Table 13, Additional file 3). Intriguingly, some of these pathways overlapped with pathways that species-specific G. ecklonigenes were involved in, such as the Dorso-ventral axis formation, $A B C$ transporters, Peroxisome and Herpes simplex virus 1 infection. The 23 KEGG pathways, including 189 genes from these contracted gene families, revealed that they were mainly classified as Tight junction, Systemic lupus erythematosus, Pathogenic Escherichia coli infection, Gap junction, Alcoholism, Pertussis, Ascorbate and aldarate metabolism, NOD-like receptor signaling pathway and so on (Supplementary Table 14, Additional file 3). Thus, it is likely that the expanded and contracted gene families played important roles in the adaptation of $G$. eckloni to the plateau water environment.

\section{WGD events and chromosome evolution in G. eckloni}

To determine the date of the G. eckloni WGD event (4R), we used inter- and intra-genomic colinear genes and calculated their synonymous substitution rates (Ks values) (Fig. 2b). The Ks distribution of homologous gene pairs in syntenic blocks indicated that the peak Ks for the G. eckloni genome was 0.10. Based on a $K$ s rate of $3.51 \times 10^{-9}$ substitutions per synonymous site per year [22], we estimated that the latest WGD (4R) happened at $14.2 \mathrm{Ma}$, which was consistent with the most recent genome duplication time of goldfish and later than the divergence time between G. eckloni and C. carpio. Similarly, an analysis of the fourfold synonymous third codon transversion (4dTv) provided additional evidence for an extra WGD event (Supplementary Fig. 8, Additional file 1).

Previous studies showed that there are several typical karyotypes in schizothoracine fish, including $2 \mathrm{n}=$ $90,92,94,98,148$, and \pm 446 [7]. Almost all species from Schizothoracinae that have been karyologically investigated are polyploid, including tetraploid and hexaploid $[6,7,9]$. The karyotype of $G$. eckloni was reported as $2 n=94$ [7]. Along with the chromosome number, which is roughly twice as large as diploid cyprinid, including $D$. rerio $(2 n=50)$, C. idellus $(2 n=48)$, Hypophthalmichthys molitrix $(2 n=48)$, and Hypophthalmichthys nobilis $(2 n=48)[23,24], G$. eckloni is likely a tetraploid species. The $D$. rerio genome allowed us to identify potential fusion and fission events that shaped the $G$. eckloni genome since the ancestral karyotype. The chromosome synteny comparison of $G$. eckloni with $D$. rerio as a reference confirmed that four chromosome fusion events occurred in G. eckloni (Fig. 1c). Notably, chromosome 22 
in $D$. rerio involved both fusion and fission events. The synteny analysis revealed a high level of collinearity between the chromosome-level genomes of $G$. eckloni and $D$. rerio, and although some were small, inter-chromosomal translocations indicated that the overall gene order in the G. eckloni genome remained very stable after its divergence from zebrafish (Fig. 1C). Previous studies corroborated that the C. carpio and $C$. auratus genome have tetraploidized due to an additional round of genome duplication (4R) and, thus, have 50 pairs of chromosomes $(2 n=4 x=100)[16,17,22,25]$, which is twice as many chromosomes as the ancestral cyprinid, $D$. rerio $(2 n=50)$. All things considered, we propose that the latest WGD (4R), chromosome fusions, fissions, and deletions were a result of the formation of karyotypes with 94 chromosomes in $G$. eckloni.

\section{Evolution of the globin gene superfamily in G. eckloni}

The globin repertoire of extant vertebrates is the product of successive genome and gene duplication events followed by differential gene retention among lineages [26-28]. Fish endemic to the QTP is comparatively well adapted to aquatic environments with low oxygen partial pressure (hypoxia), in which the globin superfamily has played an important role $[5,29,30]$. Analyses of the $G$. eckloni genome revealed 25 globin genes distributed on five chromosomes (Supplementary Table 12, Additional file 2). An $\mathrm{NJ}$ phylogenetic tree was constructed based on the alignment of all the globins from $G$. eckloni and $D$. rerio, which showed well-supported monophyletic clades of $\mathrm{Ngb}, \mathrm{Cygb}, \mathrm{Mb}, \mathrm{XGb}$, and $\mathrm{a}$ - and $\beta-\mathrm{Hb}$ (Fig. 3a). Sequence comparisons and phylogenetic analyses identified one $N g b$, one $M b$, one $X G b$, and two paralogous $C y g b$ genes (Cygb1 and Cygb2) in G. eckloni, which was consistent with a previous study on $D$. rerio [26] (Supplementary Fig. 9, Additional file 1). Moreover, the $H b$ gene repertoires of $G$. eckloni differed from $D$. rerio, based on the phylogenetic relationships and previous studies [31-33]. $H b$ genes expressed at the embryonic/larval and adult stages were denoted by " $\mathrm{e}$ " and "a," respectively. In the $G$. eckloni genome, we identified 11 a-Hb genes, including seven intact embryonic a-Hb (Hbae1.1, Hbae1.2, Hbae1.4, Hbae1.5, Hbae1.6, Hbae4, and Hbae5), two intact adult a-Hb (Hbaa1 and Hbaa2), six intact embryonic $\beta-H b$ (Hbbe1.1, Hbbe1.2, Hbbe1.4, Hbbe1.5, Hbbe1.6, and Hbbe3), two intact adult $\beta-H b$ (Hbba1and $H b b a 2)$, and three $a$ - and $\beta-H b$ incomplete or pseudogenes (Hbae1.3, Hbbe1.3, and Hbaa-like). Like zebrafish, all $H b$ genes of $G$. eckloni were distributed on two distinct chromosomes (Fig. 3b). Chromosome 20 contained the major globin locus with $17 \alpha$-and $\beta-H b$ genes and chromosome 05 housed the minor globin locus with three $\alpha$-and $\beta-H b$ genes. There were six duplicate copies in the Hbae1 and Hbbe1 genes, which was three more genes than in the zebrafish genome. Compared to the zebrafish genome, Hbae3, Hbbe3, and one copy of Hbba1-Hbbe1 gene pairs were lost in the $G$. eckloni genome. Phylogenetic analyses revealed that all the $\alpha$-and $\beta$-Hb genes, except the $H b a a-l i k e$ gene, in the $G$. eckloni genome appeared to have 1:1 orthologs in zebrafish (Hbae1, Hbae4, Hbae5, Hbaa1, Hbaa2, Hbbe1, $H b b e 3, H b b a 1$, and $H b b a 2)$. These findings suggested that the latest WGD (4R) and a recent small-scale gene duplication event after WGD contributed to gene duplication and deletion in the $\alpha$ - and $\beta-H b$ gene families in G. eckloni, which facilitated the adaptation of $G$. eckloni to the plateau water environment.

\section{Conclusions}


Using PacBio long-read sequencing data, Illumina short-read sequences, RNA-seq data and highthroughput chromosome conformation capture technology we have generated a high-quality draft genome assembly and annotation of the G. eckloni genome. Comparative analyses focused particularly on phylogenetic relationships, divergence time, and the expansion and contraction of gene families, together with the synteny analysis could deepen our understanding of the origin and speciation, as well as species polyploidization, and the evolution of chromosomes and the globin gene superfamily of the Schizothoracine fish endemic to the QTP. In conclusion, these results lay the foundation for future research on the evolution and ecology of the schizothoracine fish.

\section{Materials And Methods}

\section{Experimental fish and sequencing}

G. eckloni genomic DNA were extracted from the muscle samples of healthy female individuals obtained from the Native Fish Artificial Proliferation and Release Station, Xunhua, Qinghai Province, China (Supplementary Fig. 1, Additional file 1). For genome assembly, two libraries with insert sizes of $300 \mathrm{bp}$ and $20 \mathrm{~kb}$ were separately constructed using an Illumina TruSeq Nano DNA Library Prep Kit and SMRT bell Template Prep Kit. The two libraries were subsequently sequenced using an Illumina HiSeq X Ten instrument and a PacBio Sequel platform [34]. To conduct chromosome-level assembly of the G. eckloni genome, a Hi-C library was generated using the $\mathrm{Mbo}$ I restriction enzyme following previously described standard protocol with minor modifications [35].

\section{De novo assembly and evaluation}

We used the k-mer method to survey the genomic features of the G. eckloni. The k-mer count histogram was obtained from Illumina paired-end sequencing data using Jellyfish v2.99 [36]. Wtdbg2 v2.5 was used to obtain the best assembly with the highest continuity and accuracy of the genome [37]. Quiver [38] was used to polish the genome using long sequencing data followed by polishing with short reads using Pilon [39].

$\mathrm{Hi}-\mathrm{C}$ technology was applied to conduct the chromosome-level genome assembly of $\mathrm{G}$. eckloni. Clean reads sequenced from the Hi-C library were aligned to the contig-level genome with an end-to-end algorithm implemented in Bowtie v2.3.5 according to the Hi-C-Pro strategy [40, 41]. Juicer v1.6.2 and 3D de novo assembly (3D-DNA) pipelines were used to assemble the contigs into the chromosome-level genome $[42,43]$. The completeness of the genome was evaluated by analyzing single-copy orthologues, which was implemented by benchmarking universal single-copy orthologues using Benchmarking Universal Single-Copy Orthologs (BUSCO) v3.0.2 [44]. Synteny between the G. eckloni and D. rerio genomes was analyzed using TBtools v1.09854 [45].

\section{Assessment of genome assembly}


The assembled genome was evaluated by mapping reads from paired-end (PE) libraries with short insertsizes to the scaffolds using BWA-MEM v0.7.12 [46]. BUSCO v4.0.5 was used to evaluate the integrity of the $G$. eckloni genome [44]. The completeness of conserved genes in the G. eckloni genome was assessed by Core Eukaryotic Genes Mapping Approach (CEGMA) [47].

\section{Repeat annotation}

A combined strategy using homology alignments and de novo searches to identify whole-genome repeats was applied in our repeat annotation pipeline. Tandem repeats were extracted using TRF(http://tandem.bu.edu/trf/trf.html)by ab initio prediction. For homolog prediction, Repbase (http://www.girinst.org /repbase) employing RepeatMasker (http://www.repeatmasker.org/) software and its in-house scripts (RepeatProteinMask) with default parameters was used to extract repeat regions. Additionally, ab initio prediction based on the de novo repetitive elements database was conducted by LTR_FINDER (http://tlife.fudan.edu.cn/Itr_finder/), RepeatScout (http://www.repeatmasker.org/), and RepeatModeler (http://www.repeatmasker.org/RepeatModeler.html) with default parameters. Then, all repeat sequences with lengths $>100$ bp and gap ' $N$ ' $<5 \%$ were used to construct the raw transposable element (TE) library. A custom library (a combination of Repbase and our de novo TE library, which was processed by uclust to yield a non-redundant library) was supplied to RepeatMasker for DNA-level repeat identification.

\section{Annotation of protein-coding genes}

Gene predictions were conducted through a combination of homology, de novo, and transcriptome-based prediction methods. For homology-based predictions, the protein sequences of seven fish species, including Oryzias latipes, Ctenopharyngodon idellus, Ictalurus punctatus, Cyprinus carpio, Takifugu rubripes, Danio rerio, and Astyanax mexicanus, were downloaded from Ensembl. Protein sequences were aligned to the genome using TblastN v2.2.26 with an e-value of $1 \mathrm{e}^{-5}$ [48]. Then, matching proteins were aligned to homologous genome sequences for accurate spliced alignments using GeneWise v2.4.1 [49], which was subsequently used to predict gene structure of each protein region. RNA-sequencing data derived from nine tissues and blood samples were assembled using Trinity v2.1.1 [50]. To optimize genome annotation, RNA-seq reads from different tissues were aligned to genome fasta using TopHat package v2.0.11 with default parameters to identify exons region and splice positions [51]. The alignment results were then used as inputs for Cufflinks package v2.2.1 with default parameters for genome-based transcript assembly [52]. Finally, EvidenceModeler v1.1.1 was used to combine the gene models into weighted consensus gene structures with masked repetitive elements [53]. Additionally, PASA was used to update the final gene models, thereby adding information of alternatively spliced sites and untranslated regions (UTR).

The NR, SwissProt [54], InterPro [55], and Kyoto Encyclopedia of Genes and Genomes (KEGG) databases were used for the functional annotation of protein-coding genes using BLASTX and BLASTN utilities with an e-value threshold of $1 \mathrm{e}^{-5}[56]$.

\section{Non-coding RNA annotations}


The tRNAs were predicted using tRNAscan-SE (http://lowelab.ucsc.edu/tRNAscan-SE/). Because rRNAs are highly conserved, we chose relative species' rRNA sequences as references, and the rRNA sequences were predicted using BLAST. Other ncRNAs, including miRNAs and snRNAs, were identified by searching against the Rfam database with default parameters using infernal software (http://infernal.janelia.org/).

\section{Comparative genomic analyses}

The protein sequences of 13 vertebrates, including A. mexicanus, I. punctatus, D. rerio, C. carpio, C. idella, $O$. niloticus, $O$. latipes, $T$. rubripes, $G$. gallus, $H$. sapiens, $M$. musculus, $X$. tropicalis, and $P$. marinus, were downloaded from the Ensembl database (Release 98). Orthologous relationships between the genes from G. eckloni and the 13 other vertebrates were inferred through all-against-all protein sequence similarity searches using OthoMCL (http://orthomcl.org/orthomcl/). Only the longest predicted transcript per locus was retained. In the all-against-all BLASTP comparisons, a cutoff e-value of $1 \mathrm{e}^{-5}$ was used. The MCL inflation index was set to 1.5. A Venn diagram showing the shared orthologous groups (duplicated genes were counted as one) was based on the presence of a representative gene in at least one of the grouped species using a bioinformatics webtool (http://bioinformatics.psb.ugent.be/webtools/Venn/).

For each gene family, an alignment was produced using Muscle (http://www.drive5.com/muscle/), and ambiguously aligned positions were trimmed using Gblocks

(http://molevol.cmima.csic.es/castresana/Gblocks.html). The tree was inferred using RAxML v7.2.9 (http://sco.h-its.org/exelixis/software.html). The best-scoring ML tree was inferred by a rapid bootstrap algorithm and $\mathrm{ML}$ searches after performing 1000 rapid bootstrap replications. Divergence times between species were calculated using the MCMC tree(http://abacus.gene.ucl.ac.uk/software/paml.html)program implemented by PAML v4.6 [57]. The divergence times for $D$. rerio vs $C$. idella (48-75 Ma), A. mexicanus vs $C$. carpio (137-174 Ma), C. carpio vs T. rubripes (206-252 Ma), G. gallus vs $X$. tropicalis (347.6-358.3 Ma), T. rubripes vs G. gallus (413-443 Ma), and G. gallus vs P. marinus (515-646 Ma) were obtained from the TimeTree database then used to calibrate divergence dates of other nodes on the phylogenetic tree [58].

According to the divergence times and phylogenetic relationships, CAFÉ was used to analyze the expansion and constriction of gene families in the G. eckloni genome based on the gene families identified by OrthoMCL [59]. The phylogenetic tree topology and branch lengths were taken into account when inferring the significance of change in the gene family size of each branch. Enrichment analyses based on the Gene Ontology (GO) and KEGG annotations were performed to identify the functional implications of expanded and contracted genes (Fisher's exact test, adjusted $p$-value $<0.05$ ).

\section{Abbreviations}

QTP: Qinghai-Tibetan plateau; Ma: Million years ago; WGD: whole-genome duplication; 4R WGD: fourth whole-genome duplication; $\mathrm{Hi}-\mathrm{C}$ : high-throughput chromosome conformation capture; CEGMA: Core Eukaryotic Genes Mapping Approach; BUSCO: Benchmarking Universal Single-Copy Orthologs; CDS: coding sequence; GO: Gene Ontology; KEGG: Kyoto Encyclopedia of Genes and Genomes; MRCA: most 
recent common ancestor; Hb: hemoglobin; Ngb: neuroglobin; Cygb: cytoglobin; Mb: myoglobin; XGb: globin X.

\section{Declarations}

\section{Acknowledgments}

We thank LetPub (www.letpub.com) for its linguistic assistance during the preparation of this manuscript.

\section{Data Accessibility}

All raw data have been deposited into the CNGB Sequence Archive (CNSA) of China National GeneBank DataBase (CNGBdb) with a Project accession CNP0002087 and Sample accession CNS0401290. The PacBio long-read sequencing data, Illumina short-read sequencing data, $\mathrm{Hi}-\mathrm{C}$ sequencing data and transcriptome data are also available under Accession no. CNX0335337- CNX0335353. The genome assembly is available under the Accession no. CNA0035945. The annotation files can be downloaded from CNSA of CNGBdb (https://ftp.cngb.org/pub/CNSA/data3/CNP0002087/CNS0401290/CNA0035945/).

\section{Ethics approval and consent to participate}

This study conformed to the Guidelines for the Care and Use of Experimental Animals established by the Ministry of Science and Technology of the People's Republic of China (Approved number: 2006-398). The research protocol was reviewed and approved by the Ethical Committee of Qinghai University. The study and all methods were carried out in accordance with relevant guidelines and regulations and in compliance with the ARRIVE guidelines (https://arriveguidelines.org/).

\section{References}

1. Chen YF, Cao WY: Schizothoracinae. In: Fauna Sinica, Osteichthyes, Cypriniformes III. Edited by Yue PQ. Beijing: Science Press; 2000: 273-390.

2. Wu YF, Wu CZ: The fishes of the Qinghai - Xizang plateau. Chengdu: Science and Technology Press; 1991.

3. Qi D, Chao Y, Guo S, Zhao L, Li T, Wei F, Zhao X: Convergent, parallel and correlated evolution of trophic morphologies in the subfamily schizothoracinae from the Qinghai-Tibetan plateau. PLOS One 2012, 7(3):e34070.

4. Qi D, Chao Y, Wu R, Xia M, Chen Q, Zheng Z: Transcriptome Analysis Provides Insights Into the Adaptive Responses to Hypoxia of a Schizothoracine Fish (Gymnocypris eckloni). Front Physiol 2018, 9:1326. 
5. Xia M, Chao Y, Jia J, Li C, Kong Q, Zhao Y, Guo S, Qi D: Changes of hemoglobin expression in response to hypoxia in a Tibetan schizothoracine fish, Schizopygopsis pylzovi. J Comp Physiol $B$ 2016, 186(8):1033-1043.

6. Zan RG, Liu WG, Song Z: Tetraploid-hexaploid relationship in Schizothoracinae. Acta Genet Sin 1985, 12(2):137-142.

7. Yu XY, Li YC, Zhou T: Karyotype studies of cyprinid fishes in China -Comparative study of the karyotypes of 8 species of schizothoracine fishes. Journal of Wuhan University 1990, 2:97-104.

8. Yang S, Yan T, Zhao LL, Fu HM, Xiao Q, Pu S, Du ZJ, He Z, Yan TM: Morphogenesis of blood cell lineages in Ya-fish (Schizothorax prenanti). Chinese Journal of Zoology 2015, 50(2):231-242.

9. Dai Y, Han H: Karyological analysis of two species in the subfamily schizothoracinae (Cypriniformes: Cyprinidae) from China, with notes on karyotype evolution in schizothoracinae. Turkish Journal of Fisheries and Aquatic Sciences 2018, 18(1):175-186.

10. Xiao S, Mou Z, Fan D, Zhou H, Zou M, Zou Y, Zhou C, Yang R, Liu J, Zhu S et al: Genome of Tetraploid Fish Schizothorax o'connori Provides Insights into Early Re-diploidization and High-Altitude Adaptation. iScience 2020, 23(9):101497.

11. Qi D, Chao Y, Liang J, Gao Q, Wu R, Mather I, Zhao Y, Chen Q: Adaptive evolution of interferon regulatory factors is not correlated with body scale reduction or loss in schizothoracine fish. Fish Shellfish Immunol 2018, 73:145-151.

12. Qi D, Chao Y, Zhang C, Wang Z, Wang W, Chen Q, Zheng Z, Zhang Z: Duplication of toll-like receptor 22 in teleost fishes. Fish Shellfish Immunol 2019, 94:752-760.

13. Chen QC, Zheng ZQ, Liu D, Wang FY, Chao Y, Zhang Z, QI DL: A new pattern of hemoglobin switching in teleost fish-study of the embryonic hemoglobin in the Schizopygopsis pylzovi. Acta Hydrobiologica Sinica 2020, 44(6):1199-1207.

14. Kasahara M, Naruse K, Sasaki S, Nakatani Y, Qu W, Ahsan B, Yamada T, Nagayasu Y, Doi K, Kasai $Y$ et al: The medaka draft genome and insights into vertebrate genome evolution. Nature 2007, 447(7145):714-719.

15. Liu Z, Liu S, Yao J, Bao L, Zhang J, Li Y, Jiang C, Sun L, Wang R, Zhang Y et al: The channel catfish genome sequence provides insights into the evolution of scale formation in teleosts. Nat Commun 2016, 7:11757.

16. Xu P, Zhang X, Wang X, Li J, Liu G, Kuang Y, Xu J, Zheng X, Ren L, Wang G et al: Genome sequence and genetic diversity of the common carp, Cyprinus carpio. Nat Genet 2014, 46(11):1212-1219.

17. Chen Z, Omori Y, Koren S, Shirokiya T, Kuroda T, Miyamoto A, Wada H, Fujiyama A, Toyoda A, Zhang $S$ et al: De novo assembly of the goldfish (Carassius auratus) genome and the evolution of genes after whole-genome duplication. Sci Adv 2019, 5(6):eaav0547.

18. Qi DL, Guo SC, Chao Y, Kong QH, Li CZ, Xia MZ, Xie BS, Zhao K: The biogeography and phylogeny of schizothoracine fishes (Schizopygopsis) in the Qinghai-Tibetan Plateau. Zoologica Scripta 2015, 44(5):523-533. 
19. Pasco-Viel E, Yang L, Veran M, Balter V, Mayden RL, Laudet V, Viriot L: Stability versus diversity of the dentition during evolutionary radiation in cyprinine fish. Proc Biol Sci 2014, 281(1780):20132688.

20. Deng Y, Meng M, Fang J, Jiang H, Sun N, Lv W, Lei Y, Wang C, Bo J, Liu C et al: Genome of the butterfly hillstream loach provides insights into adaptations to torrential mountain stream life. Mol Ecol Resour 2021.

21. Shi Y, Li J, Li B, Yao TD, Wang SM, Li SJ, Cui ZJ, Wang FB, Pan B, Fang X et al: Uplift of the QinghaiXizang (Tibetan) Plateau and east asia environmental change during Late Cenozoic. Acta Geographica Sinica 1999, 54:10-21.

22. David L, Blum S, Feldman MW, Lavi U, Hillel J: Recent duplication of the common carp (Cyprinus carpio L.) genome as revealed by analyses of microsatellite loci. Mol Biol Evol 2003, 20(9):14251434.

23. Wang Y, Lu Y, Zhang Y, Ning Z, Li Y, Zhao Q, Lu H, Huang R, Xia X, Feng Q et al: The draft genome of the grass carp (Ctenopharyngodon idellus) provides insights into its evolution and vegetarian adaptation. Nat Genet 2015, 47(6):625-631.

24. Jian J, Yang L, Gan X, Wu B, Gao L, Zeng H, Wang X, Liang Z, Wang Y, Fang L et al: Whole genome sequencing of silver carp (Hypophthalmichthys molitrix) and bighead carp (Hypophthalmichthys nobilis) provide novel insights into their evolution and speciation. Mol Ecol Resour 2021, 21(3):912923.

25. Yang L, Wang Y, Zhang Z, He S: Comprehensive transcriptome analysis reveals accelerated genic evolution in a Tibet fish, Gymnodiptychus pachycheilus. Genome Biol Evol 2014, 7(1):251-261.

26. Hoffmann FG, Opazo JC, Storz JF: Whole-genome duplications spurred the functional diversification of the globin gene superfamily in vertebrates. Mol Biol Evol 2012, 29(1):303-312.

27. Opazo JC, Butts GT, Nery MF, Storz JF, Hoffmann FG: Whole-genome duplication and the functional diversification of teleost fish hemoglobins. Mol Biol Evol 2013, 30(1):140-153.

28. Schwarze K, Campbell KL, Hankeln T, Storz JF, Hoffmann FG, Burmester T: The globin gene repertoire of lampreys: convergent evolution of hemoglobin and myoglobin in jawed and jawless vertebrates. Mol Biol Evol 2014, 31(10):2708-2721.

29. Chao Y, Xia M, Wu R, Chen Q, Zheng Z, Qi D: Molecular characterization and expression changes of cytoglobin genes in response to hypoxia in a Tibetan schizothoracine fish, Schizopygopsis pylzovi. Fish Physiol Biochem 2018.

30. Qi D, Chao Y, Zhao Y, Xia M, Wu R: Molecular evolution of myoglobin in the Tibetan Plateau endemic schizothoracine fish (Cyprinidae, Teleostei) and tissue-specific expression changes under hypoxia. Fish Physiol Biochem 2018, 44(2):557-571.

31. Chan FY, Robinson J, Brownlie A, Shivdasani RA, Donovan A, Brugnara C, Kim J, Lau BC, Witkowska HE, Zon LI: Characterization of adult alpha- and beta-globin genes in the zebrafish. Blood 1997, 89(2):688-700.

32. Brownlie A, Hersey C, Oates AC, Paw BH, Falick AM, Witkowska HE, Flint J, Higgs D, Jessen J, Bahary $\mathrm{N}$ et al: Characterization of embryonic globin genes of the zebrafish. Dev Biol2003, 255(1):48-61. 
33. Ganis JJ, Hsia N, Trompouki E, de Jong JL, DiBiase A, Lambert JS, Jia Z, Sabo PJ, Weaver M, Sandstrom $\mathrm{R}$ et al: Zebrafish globin switching occurs in two developmental stages and is controlled by the LCR. Dev Bio/2012, 366(2):185-194.

34. Peng Y, Li H, Liu Z, Zhang C, Li K, Gong Y, Geng L, Su J, Guan X, Liu L et al: Chromosome-level genome assembly of the Arctic fox (Vulpes lagopus) using PacBio sequencing and Hi-C technology. Mol Ecol Resour 2021.

35. Belton JM, McCord RP, Gibcus JH, Naumova N, Zhan Y, Dekker J: Hi-C: a comprehensive technique to capture the conformation of genomes. Methods 2012, 58(3):268-276.

36. Marcais G, Kingsford C: A fast, lock-free approach for efficient parallel counting of occurrences of kmers. Bioinformatics 2011, 27(6):764-770.

37. Ruan J, Li H: Fast and accurate long-read assembly with wtdbg2. Nat Methods 2020, 17(2):155-158.

38. Chin CS, Alexander DH, Marks P, Klammer AA, Drake J, Heiner C, Clum A, Copeland A, Huddleston J, Eichler EE et al: Nonhybrid, finished microbial genome assemblies from long-read SMRT sequencing data. Nat Methods 2013, 10(6):563-569.

39. Walker BJ, Abeel T, Shea T, Priest M, Abouelliel A, Sakthikumar S, Cuomo CA, Zeng Q, Wortman J, Young SK et al: Pilon: an integrated tool for comprehensive microbial variant detection and genome assembly improvement. PLoS One 2014, 9(11):e112963.

40. Langmead B, Salzberg SL: Fast gapped-read alignment with Bowtie 2. Nat Methods 2012, 9(4):357359.

41. Servant N, Varoquaux N, Lajoie BR, Viara E, Chen CJ, Vert JP, Heard E, Dekker J, Barillot E: HiC-Pro: an optimized and flexible pipeline for Hi-C data processing. Genome Biol 2015, 16:259.

42. Durand NC, Shamim MS, Machol I, Rao SS, Huntley MH, Lander ES, Aiden EL: Juicer Provides a OneClick System for Analyzing Loop-Resolution Hi-C Experiments. Cell Syst 2016, 3(1):95-98.

43. Dudchenko O, Batra SS, Omer AD, Nyquist SK, Hoeger M, Durand NC, Shamim MS, Machol I, Lander ES, Aiden AP et al: De novo assembly of the Aedes aegypti genome using Hi-C yields chromosomelength scaffolds. Science 2017, 356(6333):92-95.

44. Simao FA, Waterhouse RM, loannidis P, Kriventseva EV, Zdobnov EM: BUSCO: assessing genome assembly and annotation completeness with single-copy orthologs. Bioinformatics 2015, 31(19):3210-3212.

45. Chen $\mathrm{C}$, Chen $\mathrm{H}$, Zhang Y, Thomas HR, Frank MH, He Y, Xia R: TBtools: An Integrative Toolkit Developed for Interactive Analyses of Big Biological Data. Mol Plant 2020, 13(8):1194-1202.

46. Li H, Durbin R: Fast and accurate short read alignment with Burrows-Wheeler transform. Bioinformatics 2009, 25(14):1754-1760.

47. Parra G, Bradnam K, Korf I: CEGMA: a pipeline to accurately annotate core genes in eukaryotic genomes. Bioinformatics 2007, 23(9):1061-1067.

48. Gertz EM, Yu YK, Agarwala R, Schaffer AA, Altschul SF: Composition-based statistics and translated nucleotide searches: improving the TBLASTN module of BLAST. BMC Bio/ 2006, 4:41. 
49. Birney E, Clamp M, Durbin R: GeneWise and Genomewise. Genome Res 2004, 14(5):988-995.

50. Grabherr MG, Haas BJ, Yassour M, Levin JZ, Thompson DA, Amit I, Adiconis X, Fan L, Raychowdhury $\mathrm{R}$, Zeng $\mathrm{Q}$ et al: Full-length transcriptome assembly from RNA-Seq data without a reference genome. Nat Biotechnol 2011, 29(7):644-652.

51. Kim D, Pertea G, Trapnell C, Pimentel H, Kelley R, Salzberg SL: TopHat2: accurate alignment of transcriptomes in the presence of insertions, deletions and gene fusions. Genome Biol 2013, 14(4):R36.

52. Stanke M, Keller O, Gunduz I, Hayes A, Waack S, Morgenstern B: AUGUSTUS: ab initio prediction of alternative transcripts. Nucleic Acids Res 2006, 34(Web Server issue):W435-439.

53. Haas BJ, Salzberg SL, Zhu W, Pertea M, Allen JE, Orvis J, White O, Buell CR, Wortman JR: Automated eukaryotic gene structure annotation using EVidenceModeler and the Program to Assemble Spliced Alignments. Genome Bio/ 2008, 9(1):R7.

54. UniProt Consortium T: UniProt: the universal protein knowledgebase. Nucleic Acids Res 2018, 46(5):2699.

55. Finn RD, Attwood TK, Babbitt PC, Bateman A, Bork P, Bridge AJ, Chang HY, Dosztanyi Z, El-Gebali S, Fraser $\mathrm{M}$ et al: InterPro in 2017-beyond protein family and domain annotations. Nucleic Acids Res 2017, 45(D1):D190-D199.

56. Kanehisa M, Goto S, Sato Y, Kawashima M, Furumichi M, Tanabe M: Data, information, knowledge and principle: back to metabolism in KEGG. Nucleic Acids Res 2014, 42(Database issue):D199-205.

57. Yang Z: PAML 4: phylogenetic analysis by maximum likelihood. Mol Biol Evol 2007, 24(8):15861591.

58. Kumar S, Stecher G, Suleski M, Hedges SB: TimeTree: A Resource for Timelines, Timetrees, and Divergence Times. Mol Biol Evol 2017, 34(7):1812-1819.

59. De Bie T, Cristianini N, Demuth JP, Hahn MW: CAFE: a computational tool for the study of gene family evolution. Bioinformatics 2006, 22(10):1269-1271.

\section{Figures}


(a)

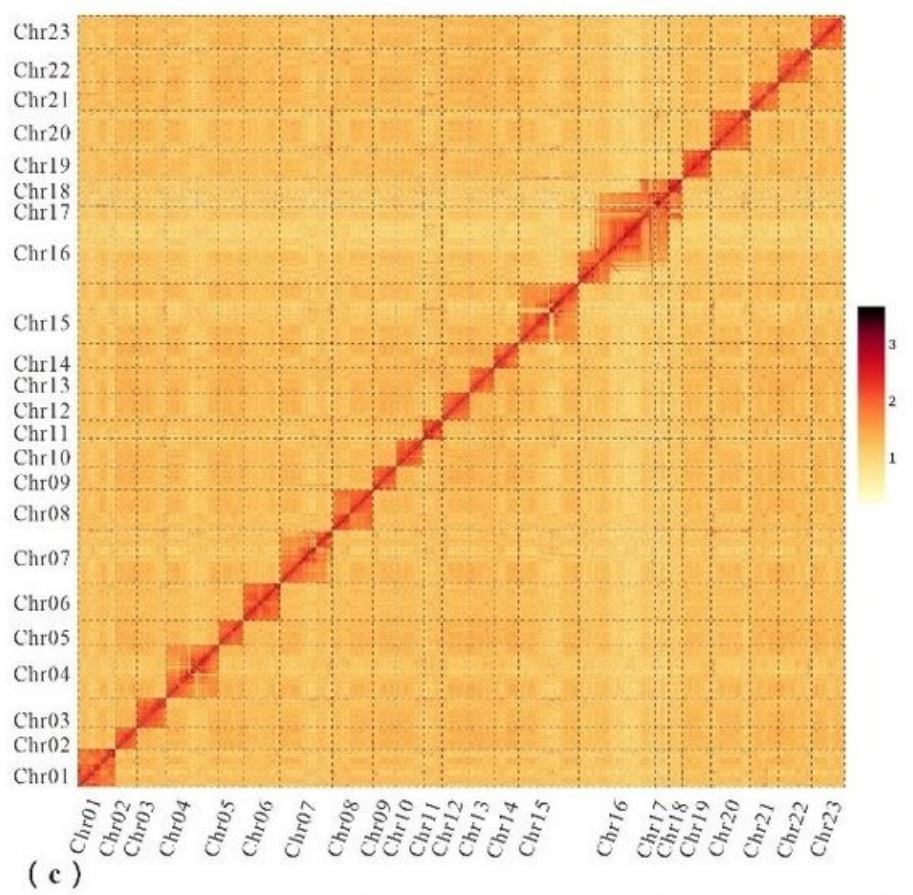

(b)

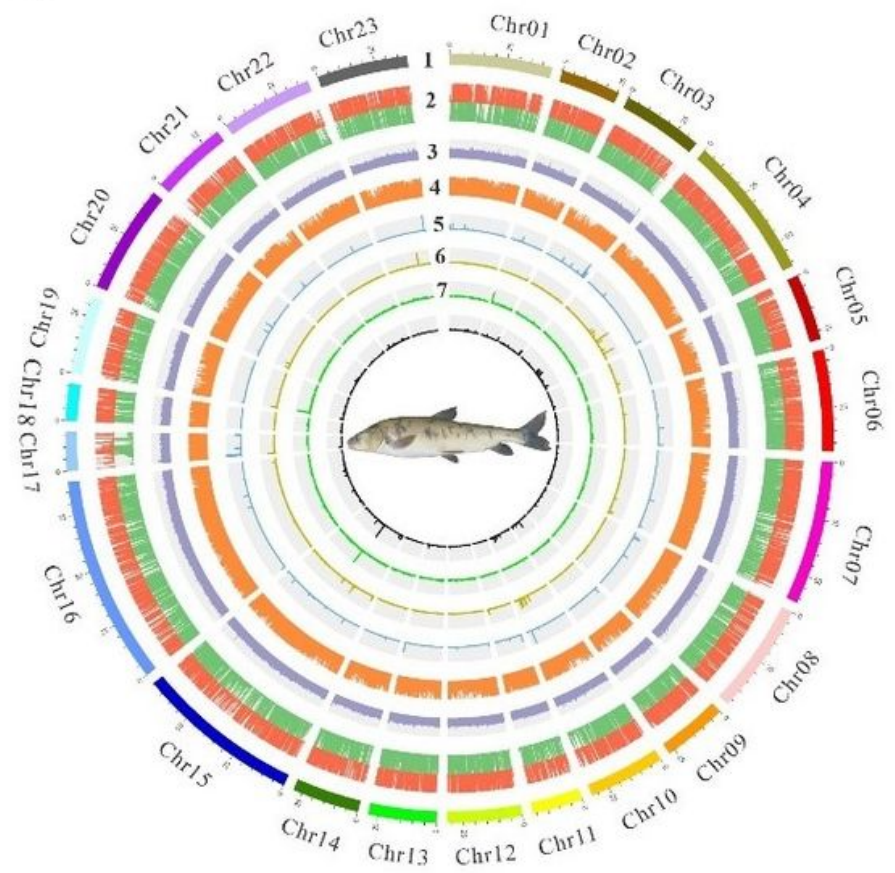

$\begin{array}{lllllllllllllllllllllllllll}\text { D. rerio } & 01 & 02 & 03 & 15 & 04 & 05 & 06 & 07 & 16 & 08 & 09 & 10 & 11 & 12 & 13 & 14 & 16 & 18 & 21 & 19 & 22 & 20 & 23 & 24 & 25\end{array}$

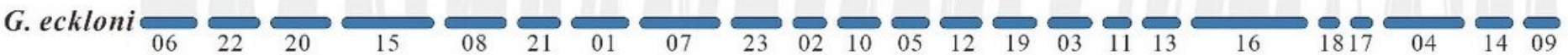

Figure 1

Characteristics of the G. eckloni genome. (a) Hi-C intra-chromosomal contact map of the G. eckloni genome assembly. (b) Circos plot of the G. eckloni genome assembly. 1) Pseudo-chromosomes; 2) gene distribution; 3) GC content; 4) repeat distribution; 5) rRNA distribution; 6) tRNA distribution; 7) miRNA distribution; 8) snRNA distribution. All data were obtained using a sliding window of $10 \mathrm{~Kb}$. (c) Synteny between $G$. eckloni and $D$. rerio genomes. 


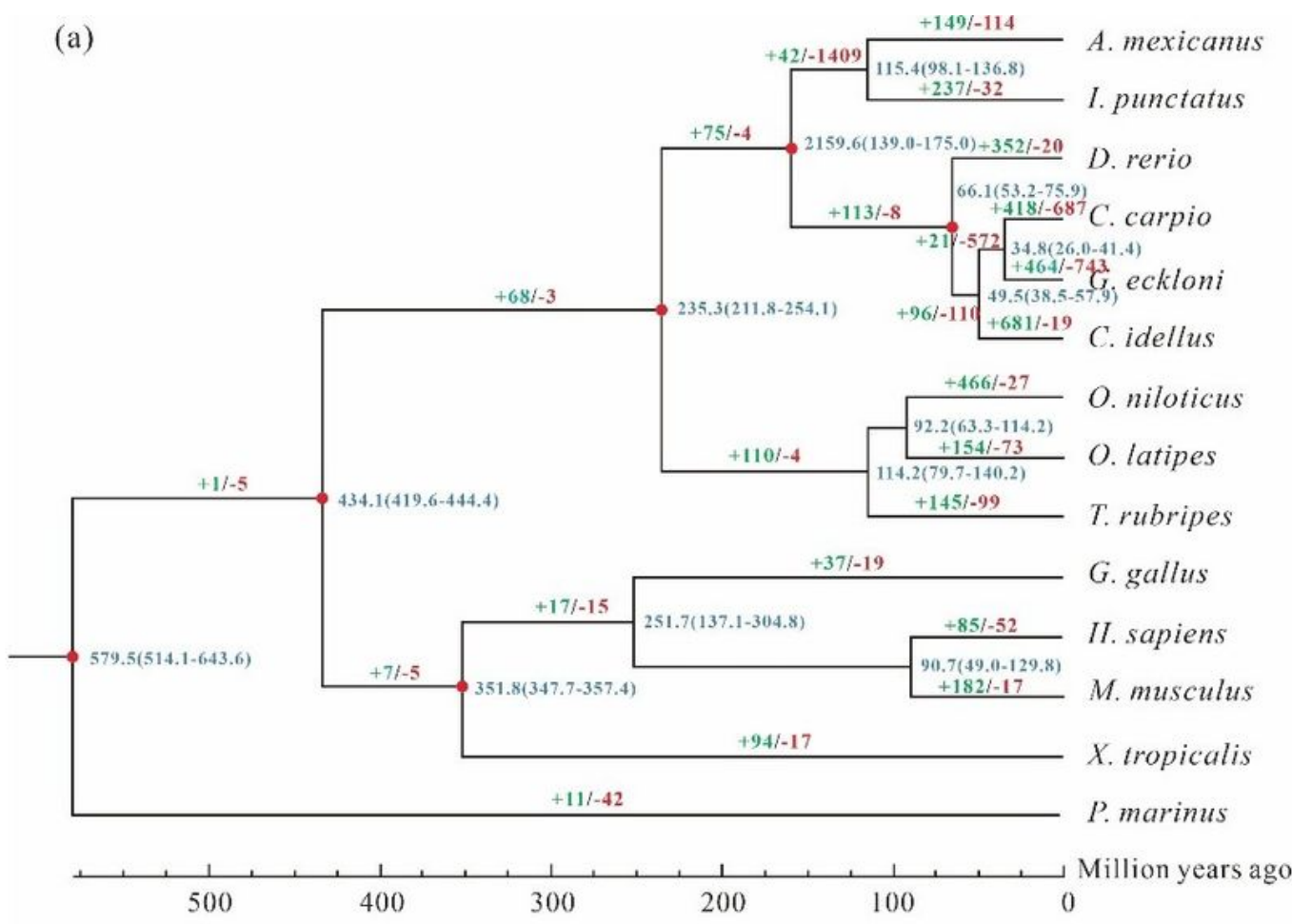

(b)

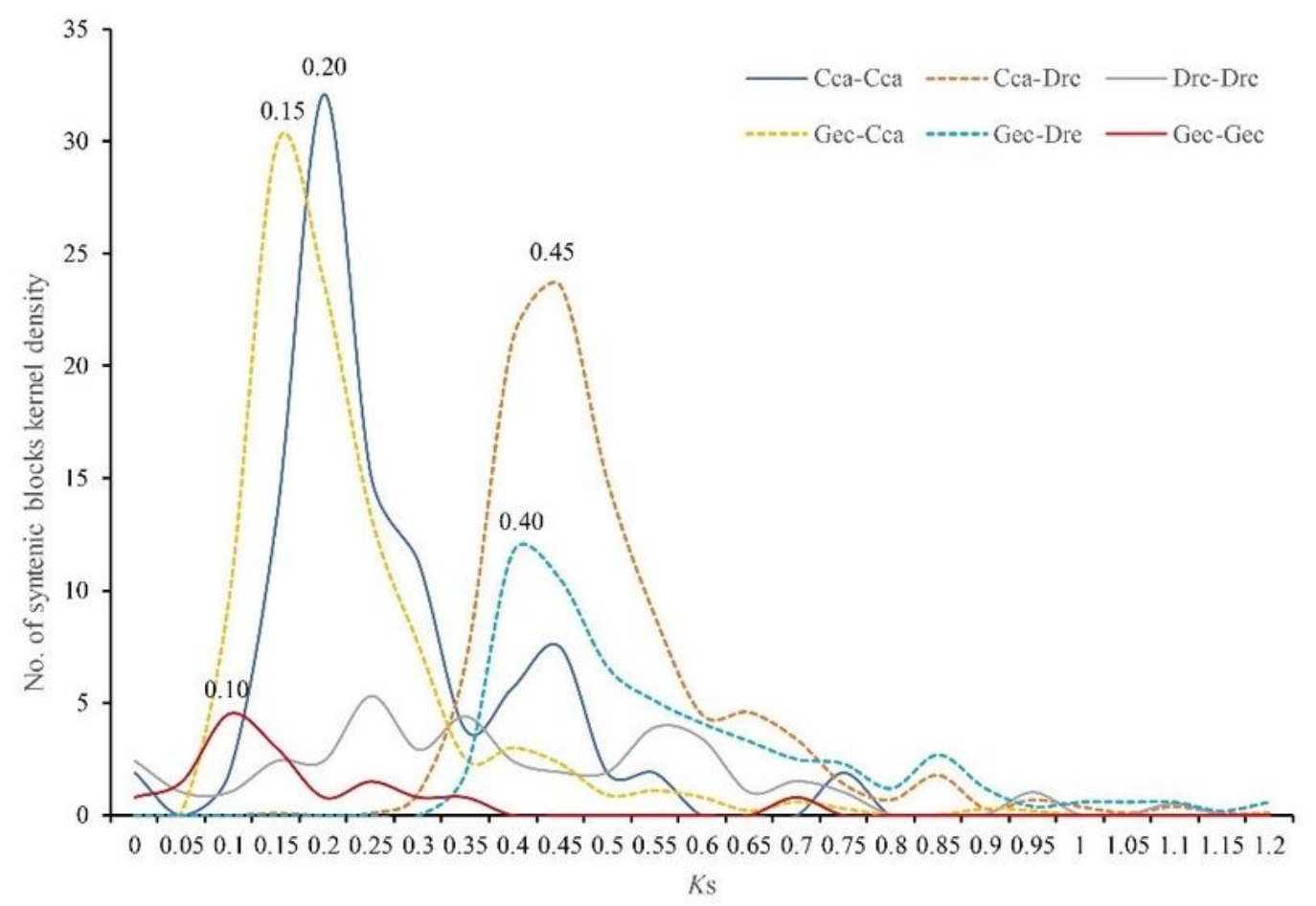

\section{Figure 2}

Evolutionary analysis of G. eckloni. (a) Phylogenetic tree based on single-copy genes from 14 species shows the estimated divergence time (blue numbers), topology and expansion (green numbers), and contraction (red numbers) of gene families. (b) Kernel density distribution of the number of synonymous substitutions per synonymous site (Ks) among colinear genes within (continuous lines) and between genomes (dashed lines). 
(a)

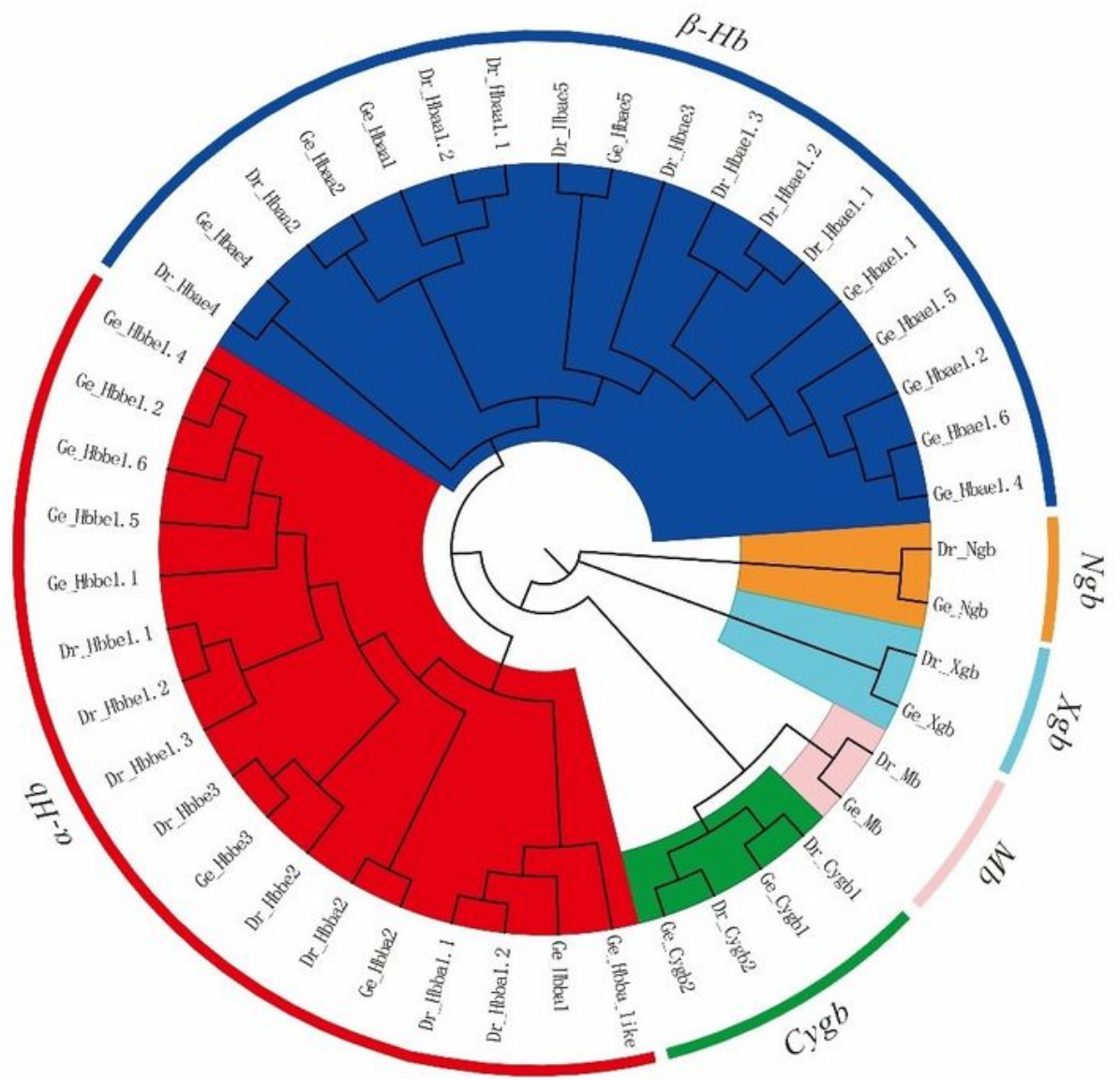

\section{Zebrafish}

$\mathrm{Chr} 3$

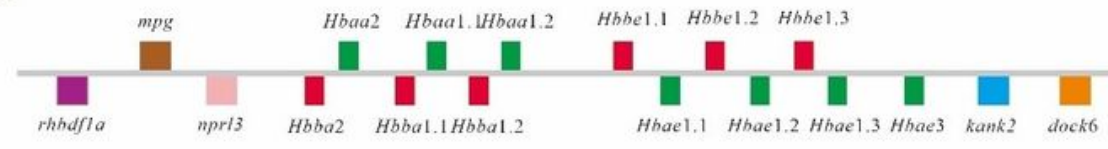

$\mathrm{Chr} 12$

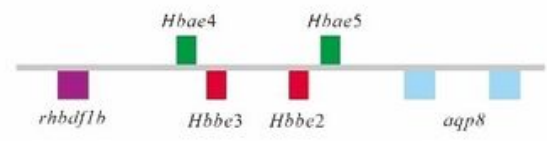

\section{G.eckloni}

Chr20

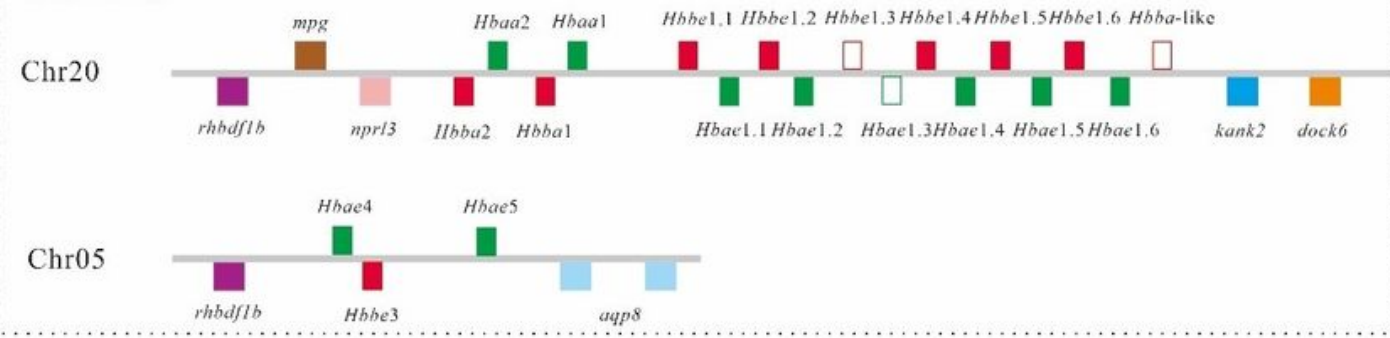

Hhbel.1 Hbbe1.2 Hbbel.3 Hbbel.4 Hbbel.5 Hbbel .6 Hbba-like

\section{Figure 3}

Evolution of the globin gene superfamily in G. eckloni. (a) Circular phylogenetic tree of the globin gene superfamily based on coding sequences. (b) Unscaled depiction of the genomic structures of the $\alpha$-and $\beta-H b$ gene families in zebrafish and G. eckloni. To facilitate comparisons, all clusters are presented in the same orientation as the zebrafish. Genes in the forward orientation are shown on top of the chromosome, while genes in the reverse orientation are shown below. 


\section{Supplementary Files}

This is a list of supplementary files associated with this preprint. Click to download.

- Additionalfile1.docx

- Additionalfile2.docx

- Additionalfile3.xlsx 